\title{
Could Peer Support Programs Be a Good Resource for Managing the Unmet Needs of Cancer Patients?
}

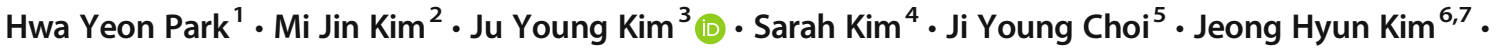 \\ Hee Yeong Jeong ${ }^{7}$
}

Published online: 8 August 2018

(C) The Author(s) 2018

\begin{abstract}
The number of cancer patients has been rapidly increasing, and while there have been wide variations, cancer survival rates also improved globally. Despite the improved survival rates, supportive care needs of cancer patients have been unmet in various domains. The current study aimed to investigate unmet needs that had potential to be managed by peer supports according to cancer trajectories. We used the comprehensive needs assessment tool in cancer (CNAT) and a modified CNAT to evaluate the unmet needs and peer support needs of cancer patients at the tertiary hospital of South Korea. Of the 402 participants, 335 $(83.3 \%)$ needed peer support. For patients who had been diagnosed with cancer for more than 5 years, the highest proportion of peer support needs to unmet supportive care was reported in information domain (92.9\%). Patients with advanced cancer reported peer support needs in the social/religious/spiritual $(84.4 \%)$ and practical domains $(81.1 \%)$. Most of stomach cancer patients needed peer supports to receive information (96.6\%). The need for peer supports in the information domain was reported highest according to longer survival period and also according to advanced cancer stages. The proportion of peer support needs in unmet supportive care varied by cancer type. Further interventional studies are needed to investigate satisfaction with peer support in specific domains.
\end{abstract}

Keywords Cancer survivorships $\cdot$ Unmet needs $\cdot$ Peer support needs $\cdot$ Mentorship $\cdot$ Peer support programs

\section{Introduction}

The number of cancer patients has been rapidly increasing worldwide as a result of the aging population and the

Hwa Yeon Park and Mi Jin Kim contributed equally to this work.

Electronic supplementary material The online version of this article (https://doi.org/10.1007/s13187-018-1399-4) contains supplementary material, which is available to authorized users.

Ju Young Kim

kkamduri@snubh.org;kkamburi@gmail.com

1 Health Promotion Center, Gachon University Gil Medical Center, 21 Namdong-daero 774beon-gil, Namdong-gu, Incheon, Republic of Korea

2 College of Nursing, Shin Han University, 30 Beolmadeul-ro 40beon-gil, Dongducheon-si, Gyeonggi-do, Republic of Korea

3 Department of Family Medicine, Seoul National University Bundang Hospital, 82 Gumi-ro 173beon-gil, Bundang-gu, Seongnam-si, Gyeonggi-do, Republic of Korea improvements in early diagnosis and treatment. It has been estimated that there are 32.6 million 5-year cancer survivors and 14 million new cancer cases worldwide each year [1], and the number of new cases is expected to increase by
4 International Healthcare Center, Seoul National University Bundang Hospital, 82 Gumi-ro 173beon-gil, Bundang-gu, Seongnam-si, Gyeonggi-do, Republic of Korea

5 Center for Cancer Information and Education, Seoul National University Bundang Hospital, 82 Gumi-ro 173beon-gil, Bundang-gu, Seongnam-si, Gyeonggi-do, Republic of Korea

6 Department of Psychiatry, Seoul National University Bundang Hospital, 82 Gumi-ro 173beon-gil, Bundang-gu, Seongnam-si, Gyeonggi-do, Republic of Korea

7 Health Promotion Center, Seoul National University Bundang Hospital, 82 Gumi-ro 173beon-gil, Bundang-gu, Seongnam-si, Gyeonggi-do, Republic of Korea 
approximately $70 \%$ over the next two decades [2]. Though there have been wide variations, globally, cancer survival has slightly improved [3]. Despite the improved survival rates, cancer patients' specific medical, psychological, informational, or social needs have reported to be largely unmet [4].

Previous studies addressing unmet needs of cancer patients mostly focused on patients with cancer stages, such as early or advanced, or with specific time periods, such as newly diagnosed cancer or long-term survivorship [5-8]. Cancer survivorship was described as time periods of survival with three phases-acute, extended, and permanent survivorship [9]. Although there are no distinctive time boundaries between the phases, patients confront to common challenges through the phases from the moment they were diagnosed with cancer to free-of-disease status [10]. Therefore, it is essential to assess unmet supportive care needs of cancer patients and examine possible resource of support according to cancer trajectories.

In this manner, social support including peer support can be a good resource in overcoming stress and has been positively associated with post-traumatic growth [11] as well as improvements in the quality of life of cancer patients [12]. One study examining the sources of supportive care in breast cancer illustrated a high need for peer support in addressing medical-psychological, social-spiritual, and sexual needs [13].

Peer support could be defined as being helped by a group of people who have the same disease and gathered together to exchange information, shared experiences, or give encouragement to each other to overcome the disease. Peer support programs which consist of cancer survivors as mentors furnish cancer patients with unique emotional and educational benefits [14]; furthermore, they represent a low cost approach and enable reallocation of some aspects of cancer care to community settings [15]. Targeting the right patients in their time of need by delivering timely informational as well as psychosocial support is essential to maximize the effect of peer support as a positive resource.

However, several review articles that explored the benefit of peer support program showed mixed results and potential mediating or moderating socio-economic factors (e.g., marital status, educational status, or economic status) on psychological outcomes $[16,17]$. Also, a detailed description regarding the peers who engaged in the one-to-one support program or recruitment process seemed limited and there might have had potential conflicts with traditional professional-based care and peer-supported program. Cost-effectiveness issues should be considered when managing the peer support program because of staff time for recruiting and supervising, development of delivery methods for peer support, or educating the support providers appropriate knowledge regarding cancer trajectory.

Unmet supportive needs can vary according to the time of cancer diagnosis $[4,18]$. At the cancer diagnosis phase, anxiety with fears was commonly identified, and during treatment phase, most unmet needs were highly reported in all domains. As time went by, many cancer patients reported persistent unmet care needs in several domains. Although unmet supportive care needs are prevalent among cancer patients, effective interventions to reduce unmet needs have been limited [19]. Though peer support might have some potential in fulfilling these unmet care needs, few studies have matched peer support domains with the highly unmet needs of cancer patients according to the stage of their cancer trajectory.

Therefore, as a basis of designing peer support programs, the aim of this study was to (1) identify unmet supportive care needs of cancer patients and (2) determine the domains for which patients considered peer support a good resource in terms of time since cancer diagnosis.

\section{Methods}

\section{Study Designs and Participants}

This cross-sectional study was conducted from January 2014 to April 2014 in a comprehensive cancer center of a tertiary hospital in South Korea. Around 600 patients were approached, 47 refused to participate and 50 did not meet inclusion criteria or exclusion criteria, so a total 503 patients were recruited. Inclusion critieria were (1) over 19 years old, (2) with diagnosis of cancer, and (3) currently undergoing evaluation for cancer stage or treatment stage or post-treatment follow-up period. Patients who had an Eastern Cooperative Oncology Group Performance Status (ECOG PS) score of 3 (capable of only limited self-care) to 5 (dead) [20] or difficulty in understanding the questionnaire were excluded.

A trained nurse conducted face-to-face interviews with visiting patients in outpatient waiting room of cancer clinics, inpatients wards, or cancer information and education center at the tertiary hospital. She explained the purpose of study and, after receiving written informed consent, asked participants to complete a questionnaire. The survey conducted in three steps. First, we assessed supportive care needs of all cancer patients using comprehensive needs assessment tool in cancer (CNAT) [21]. Second, we explained the meaning of peer support, then asked the patients if they need peer support. Third, for those who responded "yes," we examined peer support needs with modified CNAT.

To explore the unmet needs and peer support needs of cancer patients in relation to the duration of their survival, we divided them into four groups according to the time since cancer diagnosis. These groups were determined based on patients' treatment and follow-up schedules: acute survivorship, less than 3 months; transitional survivorship, 3 months2 years; extended and chronic survivorship, 2-5 years; and permanent survivorship, more than 5 years. 


\section{Measures}

\section{Supportive Care Needs}

We used CNAT to examine supportive care needs. The CNAT is self-administered questionnaire, which comprehensively measures cancer patients' needs and has been developed to cover various cancer types and all phases of cancer experiences after diagnosis. It consists of 59 items with seven domains: information (10 items), psychological care (10 items), health care staff ( 8 items), physical symptoms (12 items), hospital facilities and services ( 8 items), social/religious/spiritual support (6 items), and practical support (6 items). Internal consistency of the CNAT was reported satisfactory (Cronbach's alpha $=0.97$ for the total scales) [21].

\section{Peer Support Needs}

We described peer support as being helped by a group of people who have the same disease and gather together to exchange information, share experiences or give encouragement to each other to overcome the diseases.

To assess peer support needs, we modified some of the questions in the CNAT on basis of clinical knowledge, context of questionnaire, cultural context, and expert opinions including psychiatrist, oncologist, and oncology nurse practitioners. Through the meeting with experts, some items about unmet needs from health care staff or hospital facilities and services that could not be replaced by peer support were removed. Besides these items, a phrase of "peer support" was inserted in the questions.

For example, the items "Wished to see doctor in a quick and easy way when in need," "Wished my nurse to promptly attend to my discomfort and pain," or "Wished to have short waiting period between the reservation and the doctor's appointment" were removed. The item "Needed information or education about things that I can do at home for my health" was changed to "Needed peer support to receive information or education about things that I can do at home for my health." Another item such as "Needed help with feelings of anger, irritability, or nervousness" was also changed to "Needed peer support with feelings of anger, irritability, or nervousness."

By deleting or rephrasing items, modified CNAT comprised 40 items. The questions regarding the need for peer support consisted of five domains: information (10 items), psychological care (10 items), physical symptoms (12 items), social/religious/spiritual support (4 items), and practical support (4 items).

Patients were asked to specify their level of unmet needs or their level of needs for peer support regard to the past month using a four-point Likert scale $(0=$ no need, $1=$ low need, $2=$ moderate need, $3=$ high need). For each item, a score was given 0 , if patients had no difficulties or if the item was not relevant. If patients had needs for each question, they marked the score from 1 to 3 depending on level of need.

Since our main aim was to explore the potential utilization of peer support program in moderate-to-high level of unmet needs in cancer patients, we defined having unmet needs as at least having moderate to high needs (Likert scale more than 2). Patients who reported moderate-to-high need at least one item in a domain was considered to have needs in that domain. Also, if patients were eligible and asked to complete modified CNAT for need of peer support, same held true to have peer support needs in that domain.

\section{Statistical Analysis}

Descriptive statistics were used to describe the sociodemographic characteristics and the 10 items with the highest unmet need. Chi-squared test and Fisher's exact tests were used for categorical variables.

To assess how unmet needs and peer support needs matched, dichotomous variables were created as having moderate-to-high unmet needs (yes/no) and having moderateto-high peer support needs (yes/no). We calculated the ratio of peer support needs compared with unmet needs in each domain across the type, duration, or stage of cancer and compared the ratio using independent two population proportion test. All tests of significance were two-sided, and a $p$ value $<0.05$ was considered statistically significant. All analyses were performed using STATA version 14.0 (Stata Corp., TX, USA).

\section{Results}

\section{Participant Characteristics}

Of the 503 eligible participants, 402 completed the questionnaire. The participants' median age was 58 years (range 2289 years). More than half of the subjects were women (66.4\%), and most were married (86.0\%), and had primary cancer without recurrence $(85.1 \%)$ when the survey was conducted. Approximately a third of participants $(36.1 \%)$ were diagnosed with cancer 3 months to 2 years ago. The most common cancer was breast cancer $(36.8 \%)$, followed by colon/rectum cancer (18.7\%), thyroid cancer (11.0\%), stomach cancer $(9.0 \%)$, prostate cancer $(5.5 \%)$, and lung cancer (4.7\%). Additionally, $87.1 \%$ of subjects had received surgery to treat cancer, $61.4 \%$ chemotherapy, and $29.1 \%$ radiation therapy, with multiple answers allowed (Table 1).

\section{Description of the Most Common Unmet Needs of Cancer Patients}

Of the 402 participants, $381(94.8 \%)$ reported at least one unmet need in overall domains. The most frequently reported 
Table 1 Participant characteristics $(N=402)$

\begin{tabular}{|c|c|c|}
\hline & Number & Percent \\
\hline Age, median (range) & $58(22-89)$ & \\
\hline \multicolumn{3}{|l|}{ Age group } \\
\hline$\leq 39$ years & 41 & 10.2 \\
\hline $40-59$ years & 177 & 43.9 \\
\hline$\geq 60$ years & 184 & 45.8 \\
\hline \multicolumn{3}{|l|}{ Gender } \\
\hline Men & 135 & 33.6 \\
\hline Women & 267 & 66.4 \\
\hline \multicolumn{3}{|l|}{ Education $^{\mathrm{a}}$} \\
\hline$\leq$ High school & 229 & 61.9 \\
\hline$\geq$ College & 141 & 38.1 \\
\hline \multicolumn{3}{|l|}{ Marital status $^{\mathrm{a}}$} \\
\hline Married & 318 & 86.0 \\
\hline Separate/divorced/widowed & 27 & 7.3 \\
\hline Never married & 25 & 6.8 \\
\hline \multicolumn{3}{|l|}{ Cancer stage } \\
\hline 1 & 120 & 29.9 \\
\hline 2 & 141 & 35.1 \\
\hline 3 & 97 & 24.1 \\
\hline 4 & 44 & 11.0 \\
\hline \multicolumn{3}{|l|}{ Time since cancer diagnosis } \\
\hline$<3$ months & 68 & 16.9 \\
\hline 3 months -2 years & 146 & 36.3 \\
\hline $2-5$ years & 113 & 28.1 \\
\hline$\geq 5$ years & 75 & 18.7 \\
\hline \multicolumn{3}{|l|}{ Cancer type } \\
\hline Breast & 148 & 36.8 \\
\hline Colon/rectum & 75 & 18.7 \\
\hline Lung & 19 & 4.7 \\
\hline Thyroid & 44 & 11.0 \\
\hline Stomach & 36 & 9.0 \\
\hline Prostate & 22 & 5.5 \\
\hline Others $^{\mathrm{b}}$ & 58 & 14.4 \\
\hline \multicolumn{3}{|l|}{ Currently undergoing treatment } \\
\hline Yes & 169 & 42.0 \\
\hline No & 233 & 58.0 \\
\hline \multicolumn{3}{|l|}{ Recurrence } \\
\hline Yes & 60 & 14.9 \\
\hline No & 342 & 85.1 \\
\hline \multicolumn{3}{|l|}{ Treatment $^{\mathrm{c}}$} \\
\hline Surgery & 350 & 87.1 \\
\hline Chemotherapy & 247 & 61.4 \\
\hline Radiation & 117 & 29.1 \\
\hline
\end{tabular}

${ }^{\text {a }}$ Excluded missing data

${ }^{\mathrm{b}}$ Included esophagus, kidney, liver, pancreas, prostate, ovary, cervix, leukemia, and lymphoma

${ }^{\mathrm{c}}$ Multiple answers were allowed unmet needs in cancer patients were related to information and education. The highest unmet need among cancer patients was for information on complementary and alternative medicine (69.4\%) and education about things that patients can do at home $(69.4 \%)$. Other unmet needs in cancer patients were reported in the following order: "correct diet-which foods to eat and to avoid" (68.2\%), "information about financial support for medical expenses from government" (67.9\%), "rehabilitation medical services to help with functional recovery after treatment $(67.7 \%)$, and "my doctor to be easy, specific, and honest in his/her explanation" (66.9\%).

\section{Prevalence of Unmet Needs by Supportive Care Needs Domains}

Table 2 shows the prevalence of at least one moderate-tohigh unmet need reported by patients according to sociodemographic and clinical factors. Cancer patients had the most unmet needs in the information domain $(88.6 \%)$ and the least in the social/religious/spiritual support domain (69.4\%).

Patients with more advanced stages of cancer had current needs in health care staff $(p=0.009)$, physical symptoms $(p=0.008)$, hospital facilities and service $(p=0.03)$, and social/religious/spiritual support $(p=0.008)$. Among cancer types, patients have significant different unmet needs in all domains except for information domain, and $90 \%$ of thyroid cancer patients were likely to have most unmet needs. Cancer patients with recurrent cancer reported statistically significant higher needs in psychological care ( $p=0.004)$, physical symptoms $(p=0.004)$, hospital facilities and service $(p=0.009)$, social/religious/spiritual support $(p=0.004)$, and practical support domains $(p=0.04)$.

\section{Proportion of Peer Support Needs to Unmet Needs in Cancer Patients}

Of the 402 participants, 335 (83.3\%) needed peer support. The highest proportion of patients with peer support needs to unmet needs was in those who had been diagnosed with cancer for more than 5 years in the information domain, at $92.9 \%$. This proportion differed significantly from that of participants 3 months-2 years $(73.2 \%)$ after their diagnosis $(p=0.003)$ and those $2-5$ years $(72.8 \%)$ after their diagnosis $(p=0.003)$ (Fig. 1a).

The proportion of peer support needs to unmet needs tended to increase with cancer stage. The need for peer support in patients with stage 1 and stage 4 cancer showed a significant difference in the social/religious/spiritual support domain (55.9 vs. $84.4 \%, p=0.005)$, as well as in practical support domain (60 vs. $81.8 \%, p=0.04$ ) (Fig. 1b).

The proportion of peer support needs to unmet needs varied by cancer type. Peer support needs in the information domain 
Table 2 Moderate to high unmet needs by supportive care needs domain

\begin{tabular}{|c|c|c|c|c|c|c|c|c|c|c|c|c|c|c|}
\hline & \multicolumn{2}{|c|}{ Information } & \multicolumn{2}{|c|}{$\begin{array}{l}\text { Psychological } \\
\text { care }\end{array}$} & \multicolumn{2}{|c|}{$\begin{array}{l}\text { Health care } \\
\text { staff }\end{array}$} & \multicolumn{2}{|c|}{$\begin{array}{l}\text { Physical } \\
\text { symptoms }\end{array}$} & \multicolumn{2}{|c|}{$\begin{array}{l}\text { Hospital facilities } \\
\text { and services }\end{array}$} & \multicolumn{2}{|c|}{$\begin{array}{l}\text { Social/religious/ } \\
\text { spiritual support }\end{array}$} & \multicolumn{2}{|c|}{$\begin{array}{l}\text { Practical } \\
\text { support }\end{array}$} \\
\hline & $n$ & $\%$ & $n$ & $\%$ & $n$ & $\%$ & $n$ & $\%$ & $n$ & $\%$ & $n$ & $\%$ & $n$ & $\%$ \\
\hline Total & 356 & 88.6 & 299 & 74.4 & 308 & 76.6 & 310 & 77.1 & 326 & 81.1 & 279 & 69.4 & 292 & 72.6 \\
\hline \multicolumn{15}{|l|}{ Cancer stages $^{\mathrm{a}}$} \\
\hline 1 & 104 & 86.7 & 80 & 66.7 & 79 & 65.8 & 80 & 66.7 & 87 & 72.5 & 70 & 58.3 & 81 & 67.5 \\
\hline 2 & 126 & 89.4 & 106 & 75.2 & 112 & 79.4 & 111 & 78.7 & 117 & 83.0 & 99 & 70.2 & 104 & 73.8 \\
\hline 3 & 85 & 87.6 & 77 & 79.4 & 81 & 83.5 & 82 & 84.5 & 84 & 86.6 & 76 & 78.4 & 73 & 75.3 \\
\hline 4 & 41 & 93.2 & 36 & 81.8 & 36 & 81.8 & 37 & 84.1 & 38 & 86.4 & 34 & 77.3 & 34 & 77.3 \\
\hline \multicolumn{15}{|l|}{ Period } \\
\hline$<3$ months & 65 & 95.6 & 55 & 80.9 & 54 & 79.4 & 55 & 80.9 & 56 & 82.4 & 51 & 75.0 & 50 & 73.5 \\
\hline 3 months -2 years & 126 & 86.3 & 105 & 71.9 & 109 & 74.7 & 107 & 73.3 & 112 & 76.7 & 99 & 67.8 & 100 & 68.5 \\
\hline $2-5$ years & 99 & 87.6 & 85 & 75.2 & 86 & 76.1 & 90 & 79.6 & 94 & 83.2 & 75 & 66.4 & 84 & 74.3 \\
\hline$\geq 5$ years & 66 & 88.0 & 54 & 72.0 & 59 & 78.7 & 59 & 78.7 & 64 & 85.3 & 54 & 72.0 & 58 & 77.3 \\
\hline \multicolumn{15}{|l|}{ Cancer types ${ }^{\mathrm{b}}$} \\
\hline Breast & 130 & 87.8 & 99 & 66.9 & 100 & 67.6 & 102 & 68.9 & 105 & 70.9 & 90 & 60.8 & 98 & 66.2 \\
\hline Colon/rectum & 62 & 82.7 & 51 & 68.0 & 55 & 73.3 & 55 & 73.3 & 61 & 81.3 & 46 & 61.3 & 50 & 66.7 \\
\hline Lung & 16 & 84.2 & 15 & 78.9 & 15 & 78.9 & 14 & 73.7 & 17 & 89.5 & 14 & 73.7 & 12 & 63.2 \\
\hline Thyroid & 42 & 95.5 & 40 & 90.9 & 40 & 90.9 & 40 & 90.9 & 40 & 90.9 & 40 & 90.9 & 39 & 88.6 \\
\hline Stomach & 32 & 88.9 & 29 & 80.6 & 31 & 86.1 & 30 & 83.3 & 31 & 86.1 & 29 & 80.6 & 29 & 80.6 \\
\hline Prostate & 18 & 81.8 & 15 & 68.2 & 17 & 77.3 & 17 & 77.3 & 18 & 81.8 & 17 & 77.3 & 16 & 72.7 \\
\hline Others $^{\mathrm{c}}$ & 56 & 96.6 & 50 & 86.2 & 50 & 86.2 & 52 & 89.7 & 54 & 93.1 & 43 & 74.1 & 48 & 82.8 \\
\hline \multicolumn{15}{|l|}{ Recurrence $^{\mathrm{d}}$} \\
\hline Yes & 56 & 93.3 & 51 & 85.0 & 51 & 85.0 & 55 & 91.7 & 56 & 93.3 & 51 & 85.0 & 50 & 83.3 \\
\hline No & 300 & 87.5 & 248 & 72.3 & 257 & 74.9 & 255 & 74.3 & 270 & 78.7 & 228 & 66.5 & 242 & 70.6 \\
\hline
\end{tabular}

${ }^{a}$ Statistically significant in health care staff $(p<0.01)$, physical symptoms $(p<0.01)$, hospital facilities and services $(p<0.05)$, and social/religious/ spiritual support domains $(p<0.01)$

${ }^{\mathrm{b}}$ Statistically significant in psychological care $(p<0.01)$, health care staff $(p<0.01)$, physical symptoms $(p<0.01)$, hospital facilities and services $(p<$ $0.01)$, social/religious/spiritual support $(p<0.01)$, and practical support domains $(p<0.05)$

${ }^{\mathrm{c}}$ Included esophagus, kidney, liver, pancreas, prostate, ovary, cervix, leukemia, and lymphoma

${ }^{\mathrm{d}}$ Statistically significant in psychological care, physical symptoms, hospital facilities and services, social/religious/spiritual support, and practical support domains (all $p<0.05$ )

were significantly higher for stomach cancer (96.6\%) than for breast $(79.1 \%, p=0.03)$, colorectal $(67.3 \%, p=0.002)$, and prostate cancer $(71.4 \%, p=0.02)$. In the social/religious/spiritual support domain, peer support needs of breast cancer (78.4\%) were significantly different to colorectal $(73.9 \%$, $p=0.009)$, lung $(46.2 \%, p=0.01)$, thyroid $(59 \%, p=0.02)$, and prostate cancer $(50 \%, p=0.02)$ (Fig. 1c).

\section{Discussion}

To the best of our knowledge, this is the first study to evaluate the need for peer support in managing the unmet needs of cancer patients according to stage in cancer trajectory. Our study revealed that two thirds of the patients who reported unmet needs in comprehensive cancer care also said that they wanted peer support in information-related needs, psychological care, and physical symptoms. The longer patients had been diagnosed with cancer and the higher their stage at cancer diagnosis was, the higher their demand was for obtaining information on cancer through peer support. Additionally, patients diagnosed with advanced cancer needed peer support in the social/religious/spiritual and practical domains.

Peer support needs in the information domain were consistently high throughout all periods of patients' cancer journeys, indicating that cancer patients still wished to be well informed through peer support. Patients with more than 5 years since their cancer diagnosis, who were in the disease-free status, seemed to need more information about their health through peer support. They seldom meet their doctors or nurses after completing treatment, despite continuing to have health problems such as fear of recurrence and discomfort with being associated with the disease. In a review article, long-term 

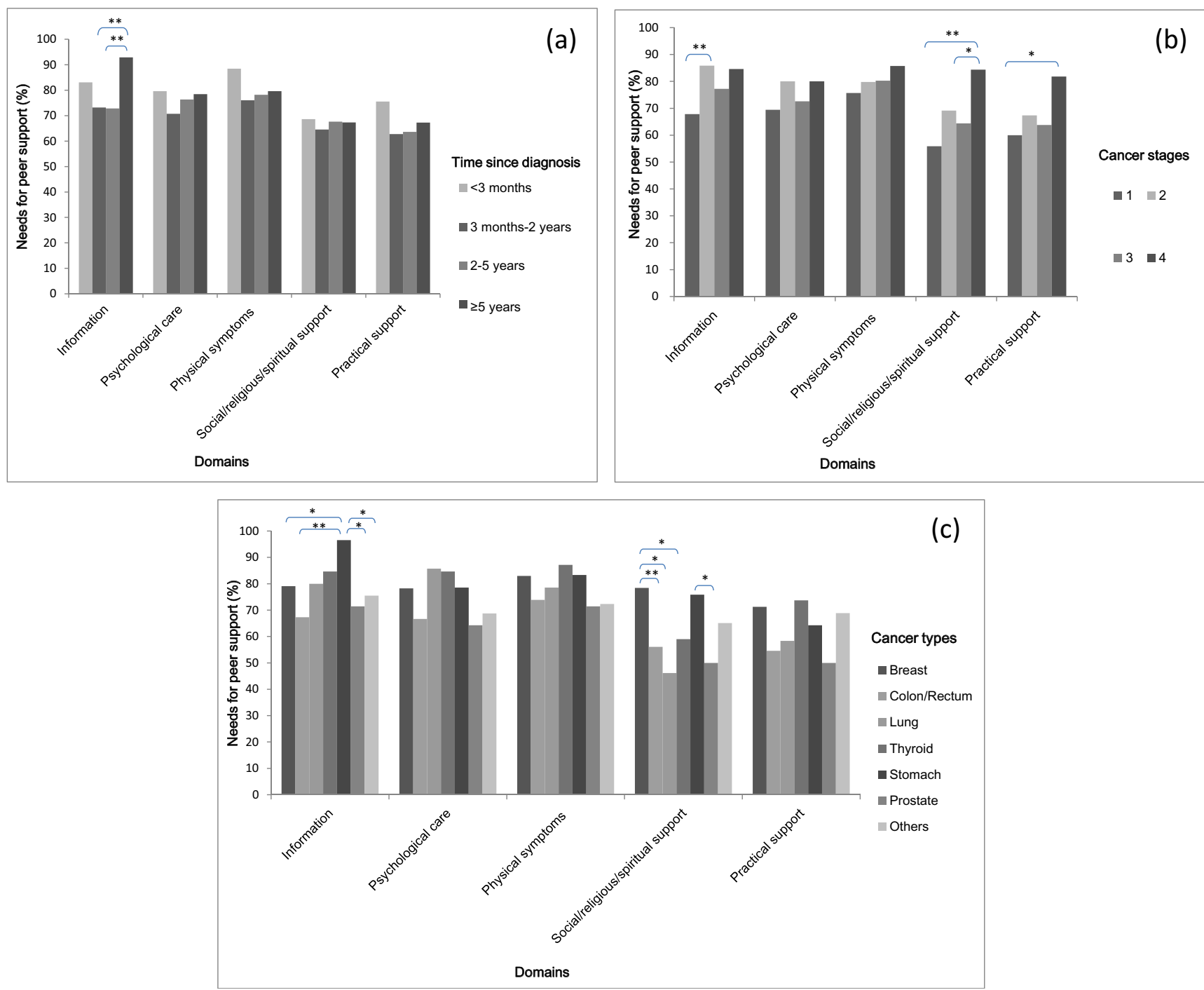

Fig. 1 Proportion of peer support needs to unmet needs according to time since cancer diagnosis (a), cancer stages (b), and cancer types (c). $* p<0.05$, $* * p<0.01$

breast cancer survivors were found to have ongoing informational and emotional needs, but they did not consistently receive help from medical staff [6]. This lack of medical support may lead them to look for complementary sources of help such as peer support.

In addition, advanced cancer patients showed a higher need for peer support in the social/religious/spiritual support and practical domains. In the literature, a review of unmet needs of patients with advanced cancer showed that unmet needs were most prevalent in the informational and psychological/ psychosocial domains in that order [5]. Coping with advanced cancer means having to live with uncertainties and doubts. Moreover, as cancer progresses, there may be more economic problem or conflict between partners, and family members or friends. In this case, peers can provide advice on relationships to patients based on their own experiences. Peer support seems to have no beneficial effects on survival of advanced cancer patients; however, it has been shown to improve psychological outcomes of them [22].

Compared with other domains, patients showed a lower need for peer support in social/religious/spiritual domains. This finding might be due to Korean cultural traits of refraining from talking about private matters with others who are not familiar with the situations. As harmony in interpersonal relationships is highly valued, people in Asian cultures tend to suppress expression of negative emotions, and their response to illness is often serenity [23]. Patients rely on people who are close to them and who can help them, and friends or family gatherings are more essential in some aspects to satisfying their needs. Among Japanese cancer patients, peer support for socialspiritual needs is sought less than support from family and friends [13]. These patients gained spiritual or social support from these closer relationships. 
However, breast cancer patients needed more peer support for social/religious/spiritual issues than patients with other types of cancer. This finding can be attributed to the characteristics of breast cancer in Korea, where the prevalence of premenopausal breast cancer and the survival rate are higher than in Western countries. The incidence of breast cancer among people in their 40s is highest in Korea and is $15 \%$ in patients under 40 years of age [24]. The 5-year survival rate of breast cancer in Korea is 91.5\% (2008-2013) [25], which is higher than the rates of $89.8 \%$ in the USA (2006-2012) [26].

Peer support programs are delivered in different formats and in numerous contexts. In a systematic review, five types of peer support were identified: one-on-one telephone, oneon-one face-to-face, group telephone, group face-to-face, and group internet support [17]. In several interventional studies, peers developed skills in listening and providing empathy, strengthened their self-care strategies under supervision from a nurse [27], and were trained to mentor through lectures, group discussion, or role play [28]. In peer counseling sessions, they received detailed treatment manuals for educating cancer patients [29]. Therefore, it is essential to consider cancer patients' specific needs and to deliver interventions according to sociodemographic and clinical factors when designing and implementing mentor programs for cancer patients.

Our study has several limitations. We focused on peer support to be concerned with cancer patients' unmet needs. Participants were not given the opportunity to indicate other types of support such as friends, family, or non-medical professors that might be helpful. However, in this study, we wanted to assess cancer survivors' role as mentors who would share their experiences with and give advices to cancer patients. Further research is needed to examine the needs of other types of support, and the effectiveness of peer support programs in fulfilling these patients' unmet needs as well. Second, peer support needs were examined using a tool derived from a validated instrument that assessed comprehensive cancer care. This tool for peer support has not been validated; however, it showed high internal consistency in this study. Finally, this study was conducted in a local hospital, and the distribution of sex and cancer type differed from that of national data. Prevalence of cancer according to the national cancer statistics were as follows: $22.0 \%$ for thyroid cancer, $15.9 \%$ for stomach cancer, $13.7 \%$ for colorectal cancer, $11.1 \%$ for breast cancer, and $4.3 \%$ for lung cancer [30]. In our study population, breast cancer accounted for one third of our study population, while thyroid cancer and stomach cancer was under-sampled compared with national data. Therefore, the study population was limited in its ability to represent the general population and may have introduced selection bias. Sociodemographic factors such as age, sex, marital status, and income level as well as clinical factors such as location of cancer, stage of disease, and tumor size can influence unmet needs in cancer patients [31]. In this regards, our study might not represent the unmet needs in male, elderly cancer survivors, and gastric cancer or thyroid cancer survivors. Future studies among representative cancer patient groups are needed.

Despite these limitations, our study results suggest that cancer patients have various unmet needs, and also need for peer support. To help them at the right time, it is important to evaluate their needs in detail and provide peer support programs based on their specific needs.

\section{Compliance with Ethical Standards}

All procedures performed in studies involving human participants were in accordance with the ethical standards of the institutional and/or national research committee and with the 1964 Helsinki declaration and its later amendments or comparable ethical standards. This study was approved by the Institutional Review Board at Seoul National University Bundang Hospital (IRB approval number B1402/239-002).

Conflicts of Interest The authors declare that they have no conflict of interest.

Open Access This article is distributed under the terms of the Creative Commons Attribution 4.0 International License (http:// creativecommons.org/licenses/by/4.0/), which permits unrestricted use, distribution, and reproduction in any medium, provided you give appropriate credit to the original author(s) and the source, provide a link to the Creative Commons license, and indicate if changes were made.

\section{References}

1. Torre LA, Bray F, Siegel RL, Ferlay J, Lortet-Tieulent J, Jemal A (2015) Global cancer statistics, 2012. CA Cancer J Clin 65(2):87108. https://doi.org/10.3322/caac.21262

2. World Health Organization (2015) Cancer fact sheet: World Health Organization; [October 23, 2016]. Available from: http://www.who. int/mediacentre/factsheets/fs297/en/

3. Allemani C, Weir HK, Carreira H, Harewood R, Spika D, Wang XS et al (2015) Global surveillance of cancer survival 1995-2009: analysis of individual data for $25,676,887$ patients from 279 population-based registries in 67 countries (CONCORD-2). Lancet 385(9972):977-1010. https://doi.org/10.1016/S01406736(14)62038-9

4. Harrison JD, Young JM, Price MA, Butow PN, Solomon MJ (2009) What are the unmet supportive care needs of people with cancer? A systematic review. Support Care Cancer 17(8):1117-1128. https:// doi.org/10.1007/s00520-009-0615-5

5. Moghaddam N, Coxon H, Nabarro S, Hardy B, Cox K (2016) Unmet care needs in people living with advanced cancer: a systematic review. Support Care Cancer 24(8):3609-3622. https://doi.org/ 10.1007/s00520-016-3221-3

6. Vivar CG, McQueen A (2005) Informational and emotional needs of long-term survivors of breast cancer. J Adv Nurs 51(5):520-528. https://doi.org/10.1111/j.1365-2648.2005.03524.x

7. Matsuyama RK, Kuhn LA, Molisani A, Wilson-Genderson MC (2013) Cancer patients' information needs the first nine months after diagnosis. Patient Educ Couns 90(1):96-102. https://doi.org/ 10.1016/j.pec.2012.09.009

8. Puts MT, Papoutsis A, Springall E, Tourangeau AE (2012) A systematic review of unmet needs of newly diagnosed older cancer 
patients undergoing active cancer treatment. Support Care Cancer 20(7):1377-1394. https://doi.org/10.1007/s00520-012-1450-7

9. Mullan F (1985) Seasons of survival: reflections of a physician with Cancer. N Engl J Med 313(4):270-273. https://doi.org/10.1056/ NEJM198507253130421

10. Stanton AL (2012) What happens now? Psychosocial Care for Cancer Survivors after Medical Treatment Completion. J Clin Oncol 30(11):1215-1220. https://doi.org/10.1200/jco.2011.39. 7406

11. McDonough MH, Sabiston CM, Wrosch C (2014) Predicting changes in posttraumatic growth and subjective well-being among breast cancer survivors: the role of social support and stress. Psycho-Oncology 23(1):114-120. https://doi.org/10.1002/pon. 3380

12. Haugland T, Wahl AK, Hofoss D, DeVon HA (2016) Association between general self-efficacy, social support, cancer-related stress and physical health-related quality of life: a path model study in patients with neuroendocrine tumors. Health Qual Life Outcomes 14:11. https://doi.org/10.1186/s12955-016-0413-y

13. Umezawa S, Fujisawa D, Fujimori M, Ogawa A, Matsushima E, Miyashita M (2015) Prevalence, associated factors and source of support concerning supportive care needs among Japanese cancer survivors. Psycho-Oncology 24(6):635-642. https://doi.org/10. 1002/pon.3702

14. Mirrielees JA, Breckheimer KR, White TA, Denure DA, Schroeder MM, Gaines ME, Wilke LG, Tevaarwerk AJ (2017) Breast Cancer survivor advocacy at a university hospital: development of a peer support program with evaluation by patients, advocates, and clinicians. J Cancer Educ 32(1):97-104. https://doi.org/10.1007/ s13187-015-0932-y

15. Ussher J, Kirsten L, Butow P, Sandoval M (2006) What do cancer support groups provide which other supportive relationships do not? The experience of peer support groups for people with cancer. Soc Sci Med 62(10):2565-2576. https://doi.org/10.1016/j. socscimed.2005.10.034

16. Meyer A, Coroiu A, Korner A (2015) One-to-one peer support in cancer care: a review of scholarship published between 2007 and 2014. Eur J Cancer Care (Engl) 24(3):299-312. https://doi.org/10. $1111 /$ ecc. 12273

17. Hoey LM, Ieropoli SC, White VM, Jefford M (2008) Systematic review of peer-support programs for people with cancer. Patient Educ Couns 70(3):315-337. https://doi.org/10. 1016/j.pec.2007.11.016

18. Sanson-Fisher R, Girgis A, Boyes A, Bonevski B, Burton L, Cook $P$ (2000) The unmet supportive care needs of patients with cancer. Supportive Care Review Group. Cancer 88(1):226-237

19. Carey M, Lambert S, Smits R, Paul C, Sanson-Fisher R, ClintonMcHarg T (2012) The unfulfilled promise: a systematic review of interventions to reduce the unmet supportive care needs of cancer patients. Supporti Care Cancer 20(2):207-219. https://doi.org/10. 1007/s00520-011-1327-1

20. Oken MM, Creech RH, Tormey DC, Horton J, Davis TE, McFadden ET et al (1982) Toxicity and response criteria of the eastern cooperative oncology group. Am J Clin Oncol 5(6):649655

21. Shim EJ, Lee KS, Park JH, Park JH (2011) Comprehensive needs assessment tool in cancer (CNAT): the development and validation. Support Care Cancer 19(12):1957-1968. https://doi.org/10.1007/ s00520-010-1037-0

22. Goodwin PJ (2005) Support groups in advanced breast cancer. Cancer 104(11 Suppl):2596-2601. https://doi.org/10.1002/cncr. 21245

23. Nilchaikovit T, Hill JM, Holland JC (1993) The effects of culture on illness behavior and medical care. Asian and American differences. Gen Hosp Psychiatry 15(1):41-50

24. Society KBC (2016) Breast Cancer Facts \& Figures 2016. Korean Breast Cancer Society, Seoul

25. Oh CM, Won YJ, Jung KW, Kong HJ, Cho H, Lee JK et al (2016) Cancer statistics in Korea: incidence, mortality, survival, and prevalence in 2013. Cancer Res Treat. 48(2):436-450. https://doi.org/ $10.4143 /$ crt.2016.089

26. Howlader N, Noone AM, Krapcho M, Miller D, Bishop K, Altekruse SF et al (2016) SEER cancer statistics review, 19752013, National Cancer Institute. Based on November 2015 SEER data submission. National Cancer Institute, Bethesda [January 6, 2017]. Available from: http://seer.cancer.gov/csr/1975_2013/

27. Schofield P, Juraskova I, Bergin R, Gough K, Mileshkin L, Krishnasamy M et al (2013) A nurse- and peer-led support program to assist women in gynaecological oncology receiving curative radiotherapy, the PeNTAGOn study (peer and nurse support trial to assist women in gynaecological oncology): study protocol for a randomised controlled trial. Trials 14:39. https://doi.org/10.1186/ 1745-6215-14-39

28. Lee R, Lee KS, Oh EG, Kim SH (2013) A randomized trial of dyadic peer support intervention for newly diagnosed breast cancer patients in Korea. Cancer Nurs 36(3):E15-E22. https://doi.org/10. 1097/NCC.0b013e3182642d7c

29. Schover LR, Rhodes MM, Baum G, Adams JH, Jenkins R, Lewis $P$ et al (2011) Sisters Peer Counseling in Reproductive Issues After Treatment (SPIRIT): a peer counseling program to improve reproductive health among African American breast cancer survivors. Cancer 117(21):4983-4992. https://doi.org/10.1002/cncr.26139

30. National Cancer Center (2015) Cancer Incidence in Korea[December 21, 2017] Available from:http://ncc.re.kr/main.ncc?uri=english/ sub04_Statistics

31. Okediji PT, Salako O, Fatiregun OO (2017) Pattern and Predictors of Unmet Supportive Care Needs in Cancer Patients. Cureus 9(5): e1234. https://doi.org/10.7759/cureus.1234 